\title{
Molecular mechanism of luteolin against inflammation based on integration of network pharmacology, transcriptomics and proteomics
}

XiuFang Huang ( $\sim 879172531 @ q q . c o m$ )

Sun Yat-Sen memory hospital of Sun Yat-Sen University https://orcid.org/0000-0003-4064-7522

Jia-Lin Zhang

southern medical university

Ai-Si Huang

Guangzhou University of Chinese Medicine

Dan-Ping Huang

Guangzhou University of Chinese Medicine

Hui-Ting Huang

Guangzhou University of Chinese Medicine

Qiong Liu

Guangzhou University of Chinese Medicine

Xiao-Hong Liu

Guangzhou University of Chinese Medicine

Hui-Li Liao

Guangzhou University of Chinese Medicine

\section{Research}

Keywords: Luteolin, Inflammation, Druggability, Transcriptomics, Proteomics, Network pharmacology

Posted Date: May 22nd, 2020

DOl: https://doi.org/10.21203/rs.3.rs-29569/v1

License: (c) (1) This work is licensed under a Creative Commons Attribution 4.0 International License.

Read Full License 


\section{Abstract}

Background: Luteolin (3', 4', 5,7-tetrahydroxyflavone), a natural flavonoid exists in various medicinal plants, has strong anti-inflammatory effect. However, anti-inflammatory mechanism of luteolin has not been fully explored. Hence, we aimed to systematically investigate druggability and anti-inflammatory mechanism of luteolin based on network pharmacology.

Methods: The absorption, distribution, metabolism, excretion, and toxicity of luteolin were evaluated by TCMSP server. Targets associated with luteolin and inflammation were collected from public databases, and the overlapping targets between luteolin and inflammation were analyzed by Draw Venn diagram. Then the protein-protein interaction network of luteolin against inflammation was constructed to get core genes. Further, gene function and pathway enrichment analysis were performed. Finally, in vitro experiment was carried out to estimate the accuracy of predicted target genes.

Results: ADME results indicated that luteolin has great potential to be developed into a drug. 226 overlapping targets (targets of luteolin against inflammation) were screened by matching 280 targets of luteolin with 9015 targets of inflammation. 9 core targets of luteolin against inflammation were identified, including MMP9, MAPK1, HSP90AA1, CASP3, ALB, EGFR, SRC, HRAS and ESR1. Gene function were mainly involved in metabolism, energy pathways and signal transduction. Pathway enrichment results suggested that metabolic pathways, pathways in cancer, PI3K-AKT signaling pathway, Ras signaling pathway and so on might be the critical pathways of luteolin against inflammation. RT-qPCR and ELISA results indicated that luteolin decreased the expression of most of core genes at protein and mRNA levels (MMP9, MAPK1, HSP90AA1, EGFR, SRC and HRAS).

Conclusions: The anti-inflammatory mechanism of luteolin were systematically investigated based on network pharmacology, RT-qPCR and ELISA. Luteolin is expounded to have great potential to be developed into a drug and target various genes and pathways to perform systematic anti-inflammatory effect.

\section{Background}

Inflammation, a defense responses to stimulation from infection and tissue damage, is related to the pathogenesis of a lot of diseases, such as sepsis, arthritis, atherosclerosis, asthma and so on ${ }^{1,2}$. Thus the effective anti-inflammatory treatment can reduce risk and prevent inflammation-related diseases and is becoming more clinically used and attracting the attention of basic researchers. However, long-term use of steroids or NSAIDs to treat inflammatory diseases can bring multiple systemic side effects. Therefore, there is an urgent need to develop new strategies for inflammatory disease.

The development of new anti-inflammatory drugs from Traditional Chinese Medicine has gradually become a research hotspot for its abundant resources and few side effects ${ }^{3}$. Chrysanthemi Flos with identified quality markers including luteolin is proved to inhibit the activation of MAPK and NF-KB in RAW 264.7 macrophages, which are involved in the progress of type 2 diabetes, atherosclerosis and cancer 4,5 . 
A clinical trial shows that Reseda luteola L. containing about 40\% luteolin decreases UVB-induced erythema, which is a inflammatory skin condition ${ }^{6,7}$. It is confirmed that flos lonicerae flavonoids isolated from Lonicerae Japonicae Flos containing luteolin decrease the expression of TNF- $a$, IL-1 $\beta$ and CRP to perform protective effect on ulcerative colitis in vivo, and HPLC-PDA assay is conducted to reveal that luteolin is one of the classified markers of Lonicerae Japonicae Flos ${ }^{8,9}$. The above researches suggest that anti-inflammatory activity of flavonoids from natural plants, especially luteolin, have been received widespread attention.

Luteolin (the structure was shown in Fig. 1) has anti-inflammatory, anti-oxidant, neuroprotective, cardioprotective, anti-diabetic, anti-microbial and anti-allergic effect as previous study ${ }^{10}$. It's worth noting that the anti-inflammatory effect of luteolin is one of its important pharmacological effect, which has been confirmed to be related to a variety of pharmacological activities. Luteolin exerts renal protection through inhibiting inflammation by activating Nrf2/ARE pathway in rats ${ }^{11}$. Luteolin can reduce inflammation to play a therapeutic role in rats with Non-alcoholic steatohepatitis ${ }^{12}$. Luteolin also can inhibit NF-KB and MAPKs activation to decrease inflammation, and thus improve insulin sensitivity in vitro $^{13}$. Theoharides et al. conduct preliminary study on clinical trial on children with Autism Spectrum Disorder, and find that treatment with luteolin significantly improves gastrointestinal and allergic symptoms in about $75 \%$ of children, and improves attention in $50 \%$ of children through performing antiinflammatory effect ${ }^{14}$. As luteolin exhibits strong anti-inflammatory activity at micromolar concentrations, luteolin and medical plants containing luteolin have become potential anti-inflammatory drugs $^{15}$. However, the anti-inflammatory mechanism of luteolin has not been systematically elucidated.

Network pharmacology combines the concepts and methods of systems biology, bioinformatics and pharmacology to explore the systematic action of Traditional Chinese Medicine or compounds, and develop new drugs ${ }^{16}$. This study intended to estimate the potential of luteolin to develop into a drug and systematically obtain the anti-inflammatory targets and mechanism of luteolin by using network pharmacology and in vitro experiment. The flowchart was shown in Fig. 2.

\section{Materials And Methods}

\section{ADME-Related properties of luteolin}

Traditional Chinese Medicine Systems Pharmacology Database and Analysis Platform (TCMSP:http://Isp.nwu.edu.cn/tcmsp.php) comprehensively provides the active compounds of all TCM recordeed in the "Pharmacopoeia of the People's Republic of China". TCMSP also contains information of absorption, distribution, metabolism, and excretion (ADME) characteristics for each compound, and druglikeness (DL) and oral bioavailability (OB) are the two most important indicators for ADME characteristics estimation of compounds ${ }^{17}$. The criteria of $\mathrm{OB} \geq 30 \%$ and $\mathrm{DL} \geq 0.18$ are important for drug development from natural plant sources ${ }^{18}$. 


\section{Collection Of Luteolin And Inflammatory Targets}

Targets of luteolin were captured by using PharmMapper (http://www.lilab-ecust.cn/p

harmmapper/submitfile.html), which is a reverse docking server for targets fishing. All the targets in PharmMapper are extracted from TargetBank, DrugBank, BindingDB and PDTD and it contains more than 7000 receptor-based pharmacophore models. PharmMapper can harveste targets of compounds through pharmacophore localization and provide the first 300 targets based on the fit score for each compound ${ }^{19}$. An sdf file for luteolin was acquired from PubChem (https://pubchem.ncbi.nlm.nih.gov/) and input to PharmMapper with default values. "Inflammation" and "anti-inflammation" performing keywords were input to Comparative Toxicogenomics Database (CTD: http://ctdbase.org/) and Genecards (https://www.genecards.org/) database to obtain targets for inflammation ${ }^{20}$. All targets were input to UniProt (http://www.uniprot.org/) to get corresponding gene symbols.

\section{Construction of Protein-protein interaction (PPI) network of luteolin against inflammation}

The targets of inflammation and luteolin were analyzed by using Draw Venn Diagram (http://bioinformatics.psb.ugent.be/Webtools/Venn/) to get the overlapping genes, which represented the targets of luteolin against inflammation. Then STRING 11.0 database (https://string-db.org/) was used to construct PPI network of luteolin against inflammation. Protein interactions with a scoring value $>0.4$ and "Homo sapiens" were selected as the high confidence basis. Cytoscape 3.7.1 software was used to visualize the PPI network of luteolin against inflammation.

\section{Core Genes Analysis}

Core genes of PPI network of luteolin against inflammation were mined by Cytohubba and MCODE plugins of Cytoscape 3.7.1 software. The parameters of CytoHubba were set as: core genes = top 10 nodes ranked by degree, Maximum Neighborhood Component (MNC), and Maximal Clique Centrality $(\mathrm{MCC})^{21}$. The parameters of MCODE were set as follows: degree cutoff $=2$, node score cutoff $=0.2, \mathrm{~K}$ score $=2$, Max deapth $=100^{22}$. Then the results of Cytohubba and MCODE analysis were analyzed by using Draw Venn Diagram to get overlapping genes, which represented the core genes.

\section{Gene Ontology (go) Enrichment Analysis}

GO, a functional system, is designed to expound gene functions and properties of gene products ${ }^{23}$. GO are mainly involved in biological process (BP), cellular component (CC) and molecular function (MF). The target genes of luteolin against inflammation were imported into FunRich software to carry out GO analysis. $P$ value区0.05 were considered to be statistically significant for $\mathrm{GO}$ enrichment ${ }^{24}$. 


\section{Kyoto Encyclopedia Of Genes And Genomes (kegg) Pathway Analysis}

KEGG pathway analysis designed to systematically analyze gene function links gene lists to higher-order functional information to receive significantly enriched biological pathways ${ }^{25}$. All targets of luteolin against inflammation were subjected to KEGG pathway analysis by using the DAVID Bioinformatics Resources 6.8 database (https://david.ncifcrf.gov/). All the results of KEGG pathway analysis with $p$ value $\mathbb{0 . 0 5}$ were selected and visualized by OmicShare tools (https://www.

omicshare.com/tools/index.php/) and the luteolin-target-pathway network was constructed by Cytoscape 3.7.126.

\section{Anti-inflammatory Effects Of Luteolin}

The RAW 264.7 macrophages were purchased from Chinese Academy of Sciences (Shanghai, China) and cultured in dulbecco's modified eagle's medium (Gibico) supplemented with $10 \%$ fetal bovine serum (Gibico), $1 \%$ streptomycin and penicillin at $37^{\circ} \mathrm{C}$ in a cell incubator containing $5 \% \mathrm{CO}_{2}$. We treated RAW 264.7 macrophages with LPS (100 ng/mL, Sigma) for $24 \mathrm{~h}$ with/without the treatment of luteolin $(5,10$ and $20 \mu \mathrm{M}$, Selleck) for $24 \mathrm{~h}$, while cells in control group were treated with medium alone for $24 \mathrm{~h} 27$.

To determine the anti-inflammatory effect of luteolin, RT-qPCR analysis was performed to confirm the effect of luteolin on the expression of core genes at mRNA level. Total RNA was collected using Trizol reagent according to the kit's instructions. RNA reverse-transcribed to CDNA was carried out using the PrimeScript RT reagent Kit (TaKaRa) with gDNA Eraser. The SYBR green PCR Master Mix was used to detect mRNA expression and samples were normalized to $\beta$-actin. 40 cycles of PCR were performed and Relative gene expression (fold change) was calculated by using $2^{-\Delta \Delta C T}$ method. Primers (Sangon Biotech) used in this study were lisetd as Table 1. Moreover, ELISA assay was performed to prove the effect of luteolin on the expression of core genes at protein level. The cell supernatants were collected to analyze by ELISA kits (R\&D Systems, Minneapolis, MN) according to the manufacturer's instructions. 
Table 1

The primers used in this study.

\begin{tabular}{|lll|}
\hline Gene name & Forward primer $\left(\mathbf{5}^{\prime} \mathbf{3}^{\prime}{ }^{\prime}\right)$ & Reverse primer $\left(\mathbf{5}^{\prime} \mathbf{3}^{\prime} \mathbf{)}\right.$ \\
\hline MAPK1 & TCTCCTCTGTGTTGTCCTCCTTCC & GGCTGCCGCTCGACTTATGC \\
\hline EGFR & TCCTGATTGGTGCTGTGCGATTC & CAACTGCTCGGATGGCTCTGTAAG \\
\hline HRAS & AGGTGCCGACGGAAGGAAGG & GCGAAGGTCTTGGTCACATCTGTC \\
\hline HSP90AA1 & AGAGACCACCACCACCACCAC & TGCCTGCCTCCACTCCTTCC \\
\hline SRC & TCACCGCCTCACTACCGTATGTC & CATCCACACCTCTCCGAAGCAAC \\
\hline -actin & CTACCTCATGAAGATCCTGACC & CACAGCTTCTCTTTGATGTCAC \\
\hline
\end{tabular}

\section{Statistical analysis}

Statistical analysis was performed using the SPSS 22.0 software. All values were presented as Mean \pm SD. One-way analysis of variance followed by least significant difference test were used for comparison. $P<0.05$ was considered statistically significant.

\section{Results}

\section{ADME-related characteristics of luteolin}

ADME characteristics of luteolin were studied in depth by TCMSP, such as OB, DL, Caco-2, BBB and Lipinski's rule of five (including MW, AlogP, TPSA, Hdon and Hacc). DL and OB are the two most important indicators for ADME characteristics estimation of compounds. Notably, luteolin was satisfied with both $\mathrm{DL} \geq 0.18$ and $\mathrm{OB} \geq 30 \%$ (Table 2).

Table 2

Pharmacological and molecular properties of luteolin.

\begin{tabular}{|llllllllll|}
\hline Name & MW & AlogP & Hdon & Hacc & OB (\%) & Caco-2 & BBB & DL & TPSA \\
\hline Luteolin & 286.25 & 2.07 & 4 & 6 & 36.16 & 0.19 & -0.84 & 0.25 & 111.13 \\
\hline
\end{tabular}

\section{Identify the assayed targets of luteolin and inflammation}

As shown in Fig. 3A and Fig. 3B, all of 280 targets of luteolin (excluded 20 targets without corresponding gene symbols) and 9015 targets related to inflammation were respectively obtained from PharmMapper, CTD and GeneCards database. The results of Draw Venn diagram suggested that 226 overlapping targets 
were screened by matching 280 targets of luteolin with 9015 targets of inflammation (Fig. 4A). It is worth noting that 226 overlapping targets represented the targets for luteolin against inflammation.

\section{PPI network construction and core genes of luteolin against inflammation verification}

Nodes and edges represented targets and interactions of targets in PPI network, respectively. STRING database showed that PPI network had 226 nodes and 2180 edges. The circles represented the targets of luteolin against inflammation and the edges represented the interaction between targets (Fig. 4B). The results of CytoHubba analysis obtained 9 core genes, including MMP9, MAPK1, HSP90AA1, CASP3, ALB, EGFR, SRC, HRAS and ESR1 (Fig. 5A). The results of MCODE obtained the top 2 most significant modules with scores of 15.647 and 10 (Fig. 5B). Convincingly, module 1 and module 2 comprised 9 core genes obtained from CytoHubba analysis, which further confirmed that the importance of MMP9, MAPK1, HSP90AA1, CASP3, ALB, EGFR, SRC, HRAS and ESR1 (Fig. 5B).

\section{Go Analysis For Target Genes Of Luteolin Against Inflammation}

$\mathrm{GO}$ analysis for target genes of luteolin against inflammation was performed by FunRich software. The results showed that $8 \mathrm{CC}$ enrichment items comprised cytoplasm, cytosol, exosomes, lysosome, extracellular, extracellular space, extracellular region and caveola (Fig. 6A). 9 items were enriched in MF, including transmembrane receptor protein tyrosine kinase activity, ligand-dependent nuclear receptor activity, protein-tyrosine kinase activity, protein serine/threonine kinase activity, catalytic activity, hydrolase activity, metallopeptidase activity, glutathione transferase activity and oxidoreductase activity (Fig. 6B). The study obtained $3 \mathrm{BP}$ enrichmen items that contained metabolism, energy pathways and signal transduction (Fig. 6C).

\section{Potential Molecular Pathways Of Luteolin Against Inflammation}

The results of KEGG pathway analysis revealed that 226 targets of luteolin against inflammation were mainly enriched in 99 signaling pathways $(P<0.05)$. Moreover, 31 signaling pathways visualized by Ominshare were directly involved in inflammation and might be the key mechanism of luteolin against inflammation, including FoxO signaling pathway, PI3K-Akt signaling pathway, Estrogen signaling pathway, HIF-1 signaling pathway, TNF signaling pathway, MAPK signaling pathway, AMPK signaling pathway and so on (Fig. 7A). The luteolin-target-pathway network details of 31 signaling pathways and enriched genes visualized by Cytoscape 3.7.1 were showed in Fig. 7B. 


\section{Anti-inflammatory Effect Of Luteolin}

Macrophages produce various pro-inflammatory mediators and chemokines when stimulated, so they are widely used to establish inflammatory cell models to study inflammatory diseases. RAW 264.7 macrophages, a mouse macrophage cell line, can generate multitype inflammatory factors when activated. Thus RAW 264.7 macrophages were selected to investigate the anti-inflammatory effect of luteolin in this study. The results of RT-qPCR and ELISA indicated that the expression of MAPK1, EGFR, HRAS, HSP90AA1, MMP9 and SRC in model group significantly increased compared with control group, and luteolin markedly inhibited MAPK1, EGFR, HRAS, HSP90AA1, MMP9 and SRC production at mRNA and protein levels (Fig. 8A-8F and Fig. 9A-9B).

\section{Discussion}

Inflammation plays a protective role when the body is infected or injured, but excessive inflammatory response will cause a wide range of diseases. Currently, more and more researches have confirmed that inflammation is closely related to the occurrence of various chronic or malignant diseases such as type 2 diabetes, rheumatoid arthritis, asthma, cancer and so on[1,2]. Therefore, there is an urgent need to develop comprehensive strategies for inflammatory disease. A certain amount of studies have been conducted on the anti-inflammatory effect of luteolin, and shown that luteolin exhibits significant antiinflammatory effect in a variety of cell and animal models ${ }^{28,29}$. However, the detailed and systematic molecular mechanism by which luteolin inhibits inflammation remains uncertain.

Evaluation of the ADME properties is the first step in digging for a drug. Studies have shown that one of the most important reasons for the failure of drug development are poor pharmacokinetics and toxic properties ${ }^{19}$. Early evaluation of ADME properties of drugs can significantly improve the success rate, and reduce the cost and the occurrence of drug toxicity and side effects of drug development ${ }^{19}$. Obviously, evaluation of ADME properties is of great significance to simplify and accelerate the drug discovery process. DL is designed to evaluate how "drug-like" a compound is and the potential of a compound to develop into a drug. The criterion of $D L \geq 0.18$ has been widely used to filter out compounds with undesirable $A D M E$-relatedproperties ${ }^{18}$. OB is one of the most critical parameter of oral drugs and the threshold of $\mathrm{OB} \geq 30 \%$ represents a good indicator of the promising effectiveness of drug delivery to the blood circulation ${ }^{18}$. Moreover, molecular weight $(\mathrm{MW})<500$ Daltons, the lipid-water partition coefficient $(\mathrm{ALog} P)<5$, the number of hydrogen bond donors (Hdon) $<5$ and the number of bond acceptors (Hacc) $\mathbb{1 0}$ are called "Lipinski's Rule of Five". A compound that complies with "Lipinski's Rule of Five" means that it will have better pharmacokinetic properties and higher bioavailability, and therefore more likely to become a drug ${ }^{19}$. As shown in Table 2, luteolin was satisfied with $D L \geq 0.18, O B \geq 30 \%$ and "Lipinski's Rule of Five" indicating that luteolin has great potential to be developed into a promising drug.

Target fishing is the second step in drug mining. The results of target genes of luteolin against inflammation network showed that 226 targets of luteolin against inflammation were obtained. 
Furthermore, 9 core genes were screened including MMP9, MAPK1, HSP90AA1, CASP3, ALB, EGFR, SRC, HRAS and ESR1. The main function of MMP9 is to degrade and reshape the homeostasis of the extracellular matrix, and plays a critical role in inflammatory response, tissue configuration, regulating matrix-bound growth factor and cytokine expression and cancer ${ }^{30}$. Moreover, research shows that luteolin decrease MMP9 expression to treat ischemic stroke, colon cancer and diabetes ${ }^{31,32}$. MAPK pathway is the intersection of signal pathways such as cell proliferation, inflammation, differentiation, functional synchronization, transformation and apoptosis, and MAPK pathway participates in cell proliferation, differentiation, canceration, metastasis, apoptosis and so $\mathrm{on}^{33}$. Luteolin decreases inflammation through inhibiting MAPK1 pathway and thus performs a beneficial treatment in atherosclerosis ${ }^{34}$. CASP3 is the main terminal cleaving enzyme in the process of apoptosis and activation of CASP3 causes apoptosis and inflammation ${ }^{35}$. Studies prove that luteolin can effectively increase CASP3 expression to induced apoptosis in HaCaT cells and cancer cells ${ }^{36,37}$. EGFR, a member of the epidermal growth factor receptor family, plays an important role in tumor cell proliferation, angiogenesis, tumor invasion and metastasis. Moreover, a decease in EGFR expression exerts anti-inflammatory activity in inflammatory diseases such as asthma ${ }^{38}$. It has been confirmed that luteolin inhibits the activation of EGFR to manage glioblastoma, lung cancer, pancreatic cancer and so on ${ }^{38-41}$. SRC can interact with phosphorylate STAT3, regulate TLR4-induced inflammatory response, regulate tumorigenesis of cancer cells and HIF1 $a$ expression ${ }^{42,43}$. Canarium subulatum and boerhavia diffusa $L$ with high content of luteolin perform anti-inflammatory activities by decreasing SRC expression ${ }^{44,45}$. HRAS mutations are more common in bladder cancer and head and neck cancer, and luteolin increases HRAS expression to regulate cell cycle progression, which may be involved in decreasing inflammation response ${ }^{46,47}$. ESR1 is essential for sexual development and reproductive function and also responsible for bone growth and maintaining normal functions of the cardiovascular and nervous systems. ESR1 mutations or abnormal expression are associated with tumor onset and excessive inflammatory response, and study suggests that luteolin might regulate acute inflammation in renal injury through affecting the expression of ESR $1^{48}$. It is worth noting that the CRP/ALB ratio is a relevant biomarker that reflects microvascular permeability, and CRP/ALB ratio has a strong correlation in assessing cohn's disease activity, analyzing the risk of acute myocardial infarction and predicting mortality in hemodialysis patients ${ }^{49-52}$. Furthermore, monitoring CRP and ALB levels together can better assess the prognosis of bacterial infectious diseases, and CRP/ALB ratio can be used as a new inflammatory prognostic indicator to predict outcomes in patients with hepatocellular carcinoma ${ }^{53,54}$. Interestingly, ALB was confirmed as a potential core gene in the target genes of luteolin against inflammation network. The above results suggest that MMP9, MAPK1, HSP90AA1, CASP3, ALB, EGFR, SRC, HRAS and ESR1 may be critical target genes of luteolin against inflammation.

Based on the analysis of gene function, the results revealed that luteolin may regulate the metabolism, energy pathways, signal transduction and activity of receptor protein and series of proteinase to defend inflammation. KEGG pathways suggested that the 31 critical signaling pathways might be the mechanism of luteolin against inflammation, including pathways in cancer, metabolic pathways, PI3KAKT signaling pathway, Ras signaling pathway, Rap1 signaling pathway and so on. The results of GO 
and KEGG analysis are in line with our prediction that core genes were mainly involved in antiinflammatory biological processes and signaling pathways. Finally, we used RT-qPCR and ELISA to further confirm the accuracy of predicted anti-inflammatory targets of luteolin. Surprisingly, the results of RT-qPCR and ELISA showed that luteolin markedly inhibited MAPK1, EGFR, HRAS, HSP90AA1, MMP9 and SRC mRNA production at protein and mRNA levels. However, we found that the levels of ALB, CASP3 and ESR1 did not increase significantly after LPS stimulation, which indicated that we need to further analyze the effect of luteolin on predicted targets expression in vivo and in vitro in proteomics and proteomics studies in the future.

\section{Conclusion}

The potential of luteolin to develop into a drug and the anti-inflammatory mechanism were predicted by using network pharmacology. The anti-inflammatory mechanism of luteolin were possibly related to core genes of MMP9, MAPK1, HSP90AA1, CASP3, ALB, EGFR, SRC, HRAS and ESR1. These results may help guide further research to identify targets for luteolin in various inflammatory diseases. The study also indicates that network pharmacology is a convincing approach for preliminarily identifying the potential of compounds from Traditional Chinese Medicine to develop into drugs and compound-related target genes.

\section{Abbreviations}

Not applicable.

\section{Declarations}

\section{Availability Of Data And Materials}

All data generated or analyzed during this study are included in this article.

\section{Ethics Approval And Consent To Participate}

Not applicable.

\section{Consent For Publication}

Not applicable.

\section{Competing interests}

All authors stated that they have no competing interests. 


\section{Funding}

This work is supported by National Natural Science Foundation of Guangdong, China (Grant no. 2018A030310520 and 2020A1515010589) and Science and Technology Program of Guangzhou, China (Grant no. 201904010235).

\section{Author contributions}

Xiao-Hong Liu and Hui-Li Liao designed this research. Xiu-Fang Huang and Jia-Lin Zhang participated in designing the study and wrote the paper. Ai-Si Huang and Dan-Ping Huang finished production of all data and reexamined the paper. Hui-Ting Huang and Qiong Liu finished the network analysis.

\section{Acknowledgements}

This work is supported by the Lingnan Medical Research Center of Guangzhou University of Chinese Medicine, Famous Traditional Chinese Medicine inheritance physician unit of Xiao-Hong Liu of Guangdong.

\section{Contributor Information}

Xiu-Fang Huang, Email:879172531@qq.com.

Jia-Lin Zhang, Email: 78281315@qq.com.

Ai-Si Huang, Email: 224419157@qq.com.

Dan-ping Huang, Email: hdping130@163.com.

Hui-Ting Huang, Email: 348619123@qq.com.

Qiong Liu, Email: 1423254686@qq.com.

Xiao-Hong Liu, Email: Ixhgzu@163.com

Hui-Li Liao, Email: lilitcm@163.com

\section{References}

1. Miki H, Tahara-Hanaoka S, Almeida MS, Hitomi K, Shibagaki S, Kanemaru K, et al. Allergin-1 Immunoreceptor Suppresses House Dust Mite-Induced Allergic Airway Inflammation. J Immunol. 2020. doi:10.4049/jimmunol.1900180. 
2. Pazzaglia S, Pioli C. Multifaceted Role of PARP-1 in DNA Repair and Inflammation: Pathological and Therapeutic Implications in Cancer and Non-Cancer Diseases. Cells 2019; 9 (1): doi:10.3390/cells9010041.

3. Lv H, Li Z, Xie Z, Hu X, Li H, Sun J, et al. Innovated formulation of TCM pangolin scales to develop a nova therapy of rheumatoid arthritis. Biomed Pharmacother. 2020;126:109872. doi:10.1016/j.biopha.2020.109872.

4. Ding G, Li B, Han Y, Liu A, Zhang J, Peng J, et al. A rapid integrated bioactivity evaluation system based on near-infrared spectroscopy for quality control of Flos Chrysanthemi. J Pharm Biomed Anal. 2016;131:391-9. doi:10.1016/j.jpba.2016.09.008.

5. Hedin U, Matic LP. Recent advances in therapeutic targeting of inflammation in atherosclerosis. $J$ Vasc Surg. 2019;69(3):944-51. doi:10.1016/j.jvs.2018.10.051.

6. Casetti F, Jung W, Wolfle U, Reuter J, Neumann K, Gilb B, et al. Topical application of solubilized Reseda luteola extract reduces ultraviolet B-induced inflammation in vivo. J Photochem Photobiol B. 2009;96(3):260-5. doi:10.1016/j.jphotobiol.2009.07.003.

7. Woelfle U, Simon-Haarhaus B, Merfort I, Schempp CM. Reseda luteola L. extract displays antiproliferative and pro-apoptotic activities that are related to its major flavonoids. Phytother Res. 2010;24(7):1033-6. doi:10.1002/ptr.3069.

8. Liu D, Yu X, Sun H, Zhang W, Liu G, Zhu L. Flos lonicerae flavonoids attenuate experimental ulcerative colitis in rats via suppression of NF-kappaB signaling pathway. Naunyn Schmiedebergs Arch Pharmacol. 2020. doi:10.1007/s00210-020-01814-4.

9. Zhao Y, Dou D, Guo Y, Qi Y, Li J, Jia D. Comparison of the Trace Elements and Active Components of Lonicera japonica flos and Lonicera flos Using ICP-MS and HPLC-PDA. Biol Trace Elem Res. 2018;183(2):379-88. doi:10.1007/s12011-017-1138-4.

10. Baek KS, Yi YS, Son YJ, Jeong D, Sung NY, Aravinthan A, et al. Comparison of anticancer activities of Korean Red Ginseng-derived fractions. J Ginseng Res. 2017;41(3):386-91. doi:10.1016/j.jgr.2016.11.001.

11. Albarakati AJA, Baty RS, Aljoudi AM, Habotta OA, Elmahallawy EK, Kassab RB, et al. Luteolin protects against lead acetate-induced nephrotoxicity through antioxidant, anti-inflammatory, anti-apoptotic, and Nrf2/HO-1 signaling pathways. Mol Biol Rep. 2020. doi:10.1007/s11033-020-05346-1.

12. Sagawa H, Naiki-Ito A, Kato H, Naiki T, Yamashita Y, Suzuki S, et al. Connexin 32 and luteolin play protective roles in non-alcoholic steatohepatitis development and its related hepatocarcinogenesis in rats. Carcinogenesis. 2015;36(12):1539-49. doi:10.1093/carcin/bgv143.

13. Nepali S, Son JS, Poudel B, Lee JH, Lee YM, Kim DK. Luteolin is a bioflavonoid that attenuates adipocyte-derived inflammatory responses via suppression of nuclear factor-kappaB/mitogenactivated protein kinases pathway. Pharmacogn Mag. 2015;11(43):627-35. doi:10.4103/09731296.160470.

14. Theoharides TC, Asadi S, Panagiotidou S. A case series of a luteolin formulation (NeuroProtek(R)) in children with autism spectrum disorders. Int J Immunopathol Pharmacol. 2012;25(2):317-23. 
doi:10.1177/039463201202500201.

15. Seelinger G, Merfort I, Schempp CM. Anti-oxidant, anti-inflammatory and anti-allergic activities of luteolin. Planta Med. 2008;74(14):1667-77. doi:10.1055/s-0028-1088314.

16. Zhai L, Ning ZW, Huang T, Wen B, Liao CH, Lin CY, et al. Cyclocarya paliurus Leaves Tea Improves Dyslipidemia in Diabetic Mice: A Lipidomics-Based Network Pharmacology Study. Front Pharmacol. 2018;9:973. doi:10.3389/fphar.2018.00973.

17. Liu L, Du B, Zhang H, Guo X, Zhou Z, Xiu A, et al. A network pharmacology approach to explore the mechanisms of Erxian decoction in polycystic ovary syndrome. Chin Med. 2018;13:46. doi:10.1186/s13020-018-0201-1.

18. $10.1155 / 2019 / 6272073$

Hu KX, Duan X, Han LZ, Ju HY, Wang B, Tang ZS, et al. Exploring Pharmacological Mechanisms of Xiang Ju Tablets in the Treatment of Allergic Rhinitis via a Network Pharmacology Approach. Evid Based Complement Alternat Med 2019; 2019 6272073. doi: 10.1155/2019/6272073.

19. Chen SJ, Cui MC. Systematic Understanding of the Mechanism of Salvianolic Acid A via Computational Target Fishing. Molecules 2017; 22 (4): doi:10.3390/molecules22040644.

20. Cui S, Chen S, Wu Q, Chen T, Li S. A network pharmacology approach to investigate the antiinflammatory mechanism of effective ingredients from Salvia miltiorrhiza. Int Immunopharmacol 2019; 106040. doi:10.1016/j.intimp.2019.106040.

21. Chin $\mathrm{CH}$, Chen $\mathrm{SH}, \mathrm{Wu} \mathrm{HH}, \mathrm{Ho} \mathrm{CW}, \mathrm{Ko} \mathrm{MT}$, Lin CY. cytoHubba: identifying hub objects and subnetworks from complex interactome. BMC Syst Biol. 2014;8(Suppl 4):11. doi:10.1186/1752-0509-8S4-S11.

22. $10.1155 / 2019 / 8567306$

Wang W, Liu Q, Wang Y, Piao H, Li B, Zhu Z, et al. Integration of Gene Expression Profile Data of Human Epicardial Adipose Tissue from Coronary Artery Disease to Verification of Hub Genes and Pathways. Biomed Res Int 2019; 2019 8567306. doi: 10.1155/2019/8567306.

23. Martucci D, Masseroli M, Pinciroli F. Gene ontology application to genomic functional annotation, statistical analysis and knowledge mining. Stud Health Technol Inform. 2004;102:108-31.

24. Zhao H, Shan Y, Ma Z, Yu M, Gong B. A network pharmacology approach to explore active compounds and pharmacological mechanisms of epimedium for treatment of premature ovarian insufficiency. Drug Des Devel Ther. 2019;13:2997-3007. doi:10.2147/DDDT.S207823.

25. Liang B, Li C, Zhao J. Identification of key pathways and genes in colorectal cancer using bioinformatics analysis. Med Oncol. 2016;33(10):111. doi:10.1007/s12032-016-0829-6.

26. Sun W, Chen Y, Li H, Liu H, Li J, Chen J, et al. Material basis and molecular mechanisms of Dachengqi decoction in the treatment of acute pancreatitis based on network pharmacology. Biomed Pharmacother. 2020;121:109656. doi:10.1016/j.biopha.2019.109656.

27. Rakariyatham K, Wu X, Tang Z, Han Y, Wang Q, Xiao H. Synergism between luteolin and sulforaphane in anti-inflammation. Food Funct. 2018;9(10):5115-23. doi:10.1039/c8fo01352g. 
28. Lee JK, Kim SY, Kim YS, Lee WH, Hwang DH, Lee JY. Suppression of the TRIF-dependent signaling pathway of Toll-like receptors by luteolin. Biochem Pharmacol. 2009;77(8):1391-400. doi:10.1016/j.bcp.2009.01.009.

29. Chen D, Bi A, Dong X, Jiang Y, Rui B, Liu J, et al. Luteolin exhibits anti-inflammatory effects by blocking the activity of heat shock protein 90 in macrophages. Biochem Biophys Res Commun. 2014;443(1):326-32. doi:10.1016/j.bbrc.2013.11.122.

30. Lu J, Li G, He K, Jiang W, Xu C, Li Z, et al. Luteolin exerts a marked antitumor effect in cMetoverexpressing patient-derived tumor xenograft models of gastric cancer. J Transl Med. 2015;13:42. doi:10.1186/s12967-015-0398-z.

31. Luo S, Li H, Mo Z, Lei J, Zhu L, Huang Y, et al. Connectivity map identifies luteolin as a treatment option of ischemic stroke by inhibiting MMP9 and activation of the PI3K/Akt signaling pathway. Exp Mol Med. 2019;51(3):37. doi:10.1038/s12276-019-0229-z.

32. Tsai PH, Cheng CH, Lin CY, Huang YT, Lee LT, Kandaswami CC, et al. Dietary Flavonoids Luteolin and Quercetin Suppressed Cancer Stem Cell Properties and Metastatic Potential of Isolated Prostate Cancer Cells. Anticancer Res. 2016;36(12):6367-80. doi:10.21873/anticanres.11234.

33. Javan GT, Salhotra A, Finley SJ, Soni S. Erythroblast macrophage protein (Emp): Past, present, and future. Eur J Haematol. 2018;100(1):3-9. doi:10.1111/ejh.12983.

34. Xia F, Wang C, Jin Y, Liu Q, Meng Q, Liu K, et al. Luteolin protects HUVECs from TNF-alpha-induced oxidative stress and inflammation via its effects on the Nox4/ROS-NF-kappaB and MAPK pathways. J Atheroscler Thromb. 2014;21(8):768-83. doi:10.5551/jat.23697.

35. Guo M, Chen H, Duan W, Li Z, Li Y, Ma Y, et al. FGF9 knockout in GABAergic neurons induces apoptosis and inflammation via the Fas/caspase-3 pathway in the cerebellum of mice. Brain Res Bull. 2020;154:91-101. doi:10.1016/j.brainresbull.2019.10.012.

36. Sinha A. P KS. Enhanced Induction of Apoptosis in HaCaT Cells by Luteolin Encapsulated in PEGylated Liposomes-Role of Caspase-3/Caspase-14. Appl Biochem Biotechnol. 2019;188(1):14764. doi:10.1007/s12010-018-2907-z.

37. Zhang H, Li X, Zhang Y, Luan X. Luteolin induces apoptosis by activating Fas signaling pathway at the receptor level in laryngeal squamous cell line Hep-2 cells. Eur Arch Otorhinolaryngol. 2014;271(6):1653-9. doi:10.1007/s00405-014-2903-z.

38. El-Hashim AZ, Khajah MA, Babyson RS, Renno WM, Ezeamuzie Cl, Benter IF, et al. Ang-(1-7)/ MAS1 receptor axis inhibits allergic airway inflammation via blockade of Src-mediated EGFR transactivation in a murine model of asthma. PLoS One. 2019;14(11):e0224163. doi:10.1371/journal.pone.0224163.

39. Anson DM, Wilcox RM, Huseman ED, Stump TA, Paris RL, Darkwah BO, et al. Luteolin Decreases Epidermal Growth Factor Receptor-Mediated Cell Proliferation and Induces Apoptosis in Glioblastoma Cell Lines. Basic Clin Pharmacol Toxicol. 2018;123(6):678-86. doi:10.1111/bcpt.13077. 
40. Huang X, Bhugul PA, Fan G, Ye T, Huang S, Dai S, et al. Luteolin inhibits pancreatitisinduced acinarductal metaplasia, proliferation and epithelialmesenchymal transition of acinar cells. Mol Med Rep. 2018;17(3):3681-9. doi:10.3892/mmr.2017.8327.

41. Hong Z, Cao X, Li N, Zhang Y, Lan L, Zhou Y, et al. Luteolin is effective in the non-small cell lung cancer model with L858R/T790M EGF receptor mutation and erlotinib resistance. Br J Pharmacol. 2014;171(11):2842-53. doi:10.1111/bph.12610.

42. Li W, Li Y, Qin K, Du B, Li T, Yuan H, et al. Siglec-G Deficiency Ameliorates Hyper-Inflammation and Immune Collapse in Sepsis via Regulating Src Activation. Front Immunol. 2019;10:2575. doi:10.3389/fimmu.2019.02575.

43. Fan JJ, Hsu WH, Lee KH, Chen KC, Lin CW, Lee YA, et al. Dietary Flavonoids Luteolin and Quercetin Inhibit Migration and Invasion of Squamous Carcinoma through Reduction of Src/Stat3/S100A7 Signaling. Antioxidants (Basel) 2019; 8 (11): doi:10.3390/antiox8110557.

44. Choi E, Kim MY, Cho JY. Anti-inflammatory activities of Canarium subulatum Guillaumin methanol extract operate by targeting Src and Syk in the NF-kappaB pathway. J Ethnopharmacol. 2019;238:111848. doi:10.1016/j.jep.2019.111848.

45. $10.1155 / 2015 / 967053$

Lee JO, Jeong D, Kim MY, Cho JY. ATP-Binding Pocket-Targeted Suppression of Src and Syk by Luteolin Contributes to Its Anti-Inflammatory Action. Mediators Inflamm 2015; 2015 967053. doi: 10.1155/2015/967053.

46. Kobayashi T, Nakata T, Kuzumaki T. Effect of flavonoids on cell cycle progression in prostate cancer cells. Cancer Lett. 2002;176(1):17-23. doi:10.1016/s0304-3835(01)00738-8.

47. Gupta DK, Kamranvar SA, Du J, Liu L, Johansson S. Septin and Ras regulate cytokinetic abscission in detached cells. Cell Div. 2019;14:8. doi:10.1186/s13008-019-0051-y.

48. Xu YH, Jian L, Ma XP, Lu Y, Chen P, Luo SW, et al. Gene expression profiling of human kidneys undergoing laparoscopic donor nephrectomy. JSLS. 2014;18(1):102-9. doi:10.4293/10860813X13693422519154.

49. 10.1186/2110-5820-2-S1-S1

Cordemans C, De Laet I, Van Regenmortel N, Schoonheydt K, Dits H, Huber W, et al. Fluid management in critically ill patients: the role of extravascular lung water, abdominal hypertension, capillary leak, and fluid balance. Ann Intensive Care 2012; 2 (Suppl 1 Diagnosis and management of intra-abdominal hyperten): S1. doi: 10.1186/2110-5820-2-S1-S1.

50. Qin G, Tu J, Liu L, Luo L, Wu J, Tao L, et al. Serum Albumin and C-Reactive Protein/Albumin Ratio Are Useful Biomarkers of Crohn's Disease Activity. Med Sci Monit. 2016;22:4393-400. doi:10.12659/msm.897460.

51. Takahashi R, Ito Y, Takahashi H, Ishii H, Kasuga H, Mizuno M, et al. Combined values of serum albumin, C-reactive protein and body mass index at dialysis initiation accurately predicts long-term mortality. Am J Nephrol. 2012;36(2):136-43. doi:10.1159/000339940. 
52. Djousse L, Rothman KJ, Cupples LA, Levy D, Ellison RC. Serum albumin and risk of myocardial infarction and all-cause mortality in the Framingham Offspring Study. Circulation. 2002;106(23):2919-24. doi:10.1161/01.cir.0000042673.07632.76.

53. Kinoshita A, Onoda H, Imai N, Iwaku A, Oishi M, Tanaka K, et al. The C-reactive protein/albumin ratio, a novel inflammation-based prognostic score, predicts outcomes in patients with hepatocellular carcinoma. Ann Surg Oncol. 2015;22(3):803-10. doi:10.1245/s10434-014-4048-0.

54. Edgar JD, Gabriel V, Gallimore JR, McMillan SA, Grant J. A prospective study of the sensitivity, specificity and diagnostic performance of soluble intercellular adhesion molecule 1, highly sensitive C-reactive protein, soluble E-selectin and serum amyloid $A$ in the diagnosis of neonatal infection. BMC Pediatr. 2010;10:22. doi:10.1186/1471-2431-10-22.

\section{Figures}

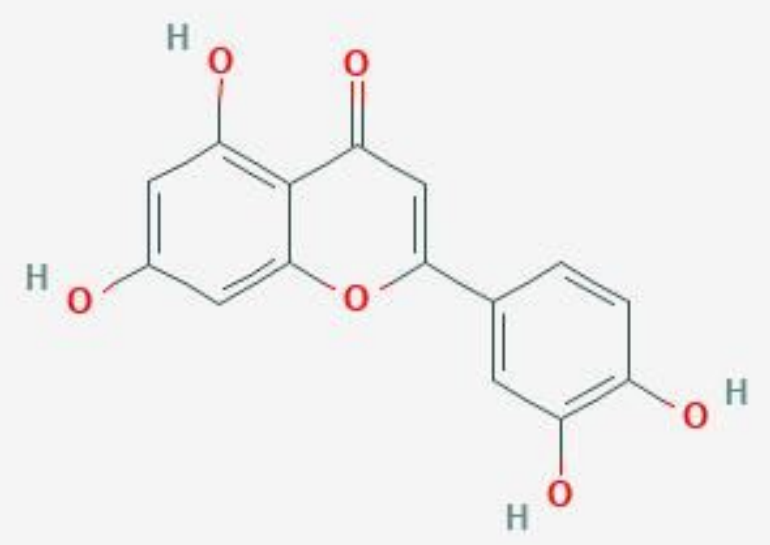

\section{Figure 1}

Structure of luteolin (PubChem CID: 5280445). 


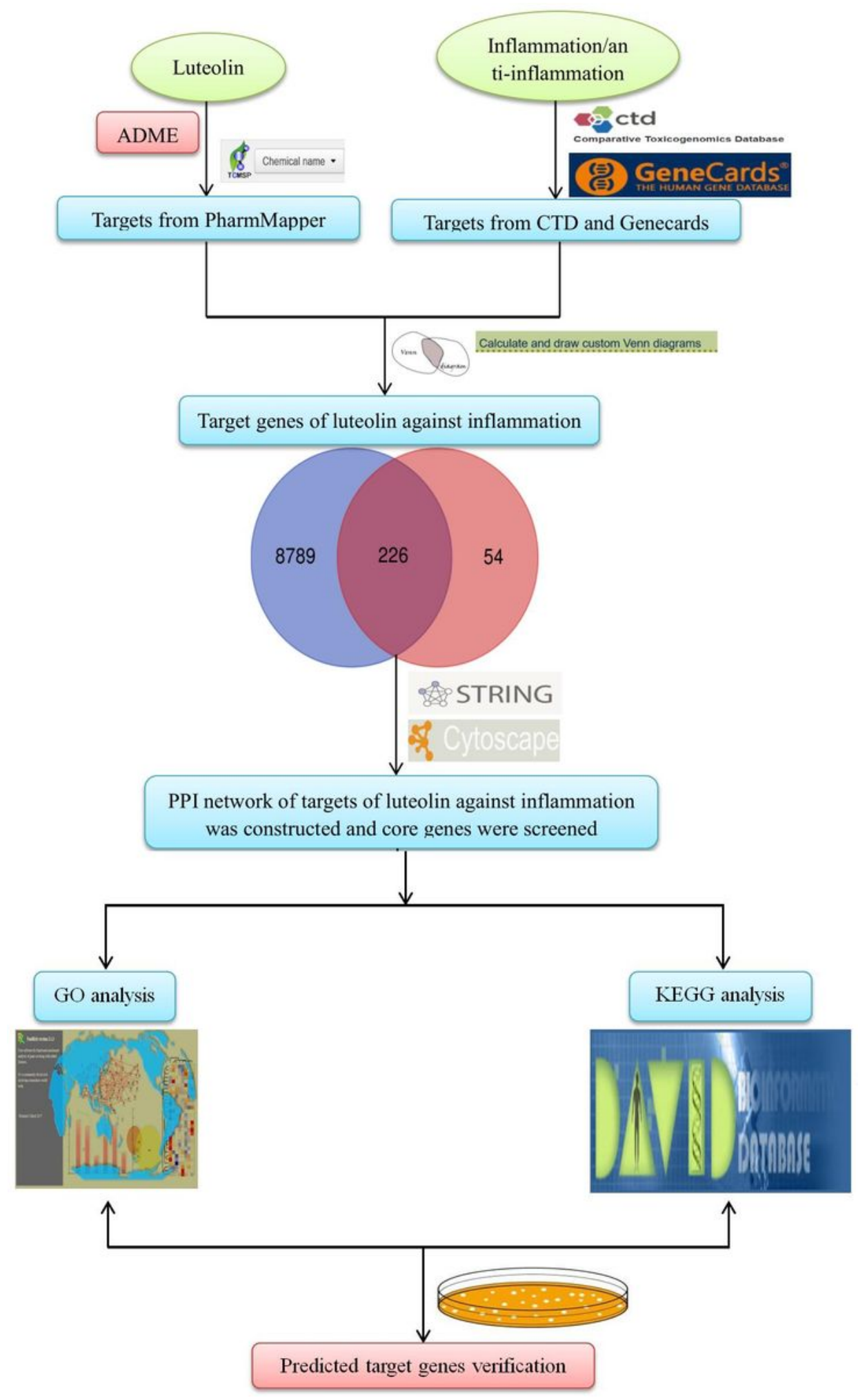

\section{Figure 2}

Workflow of network pharmacology and molecular docking analysis. 
A

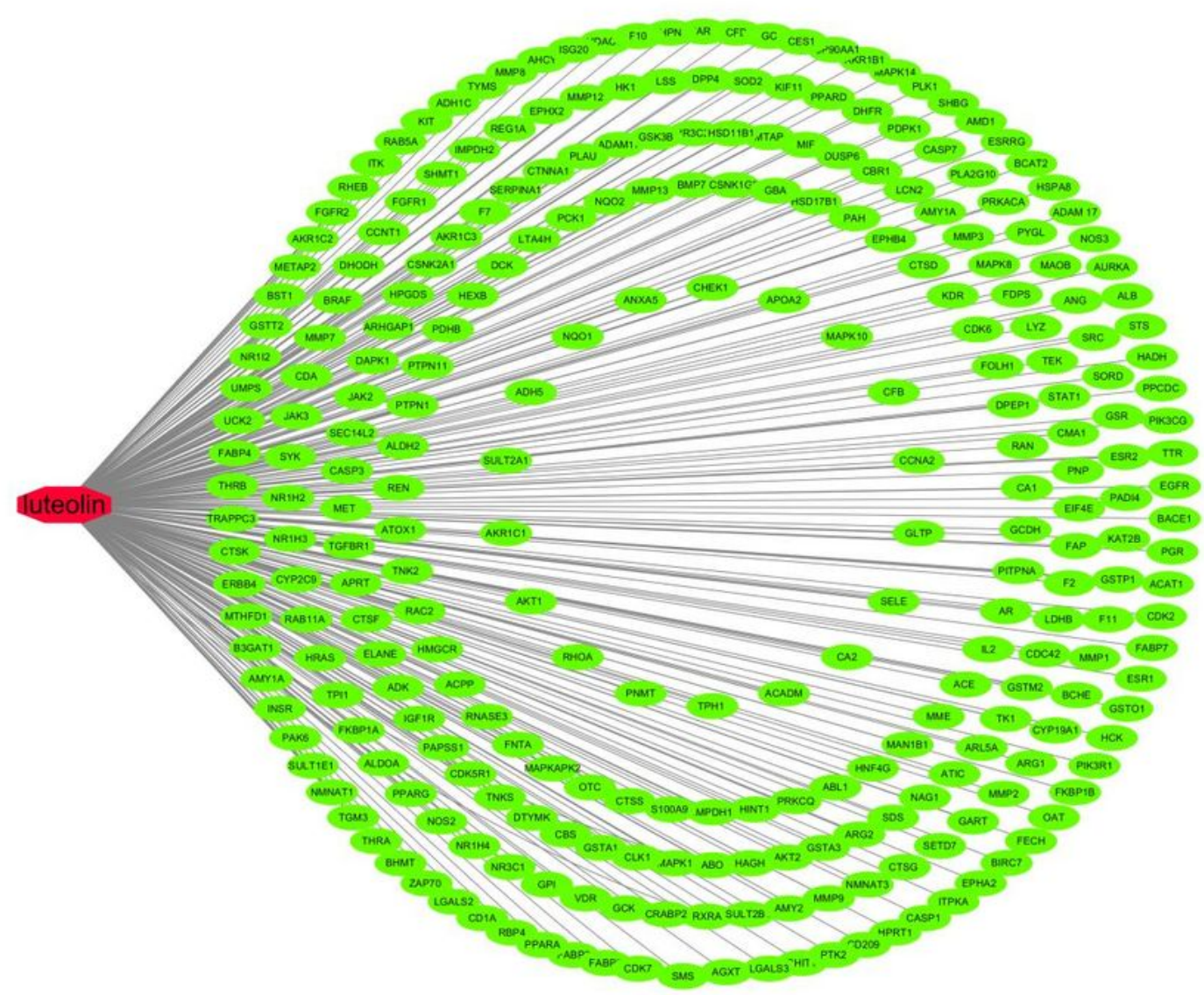

B

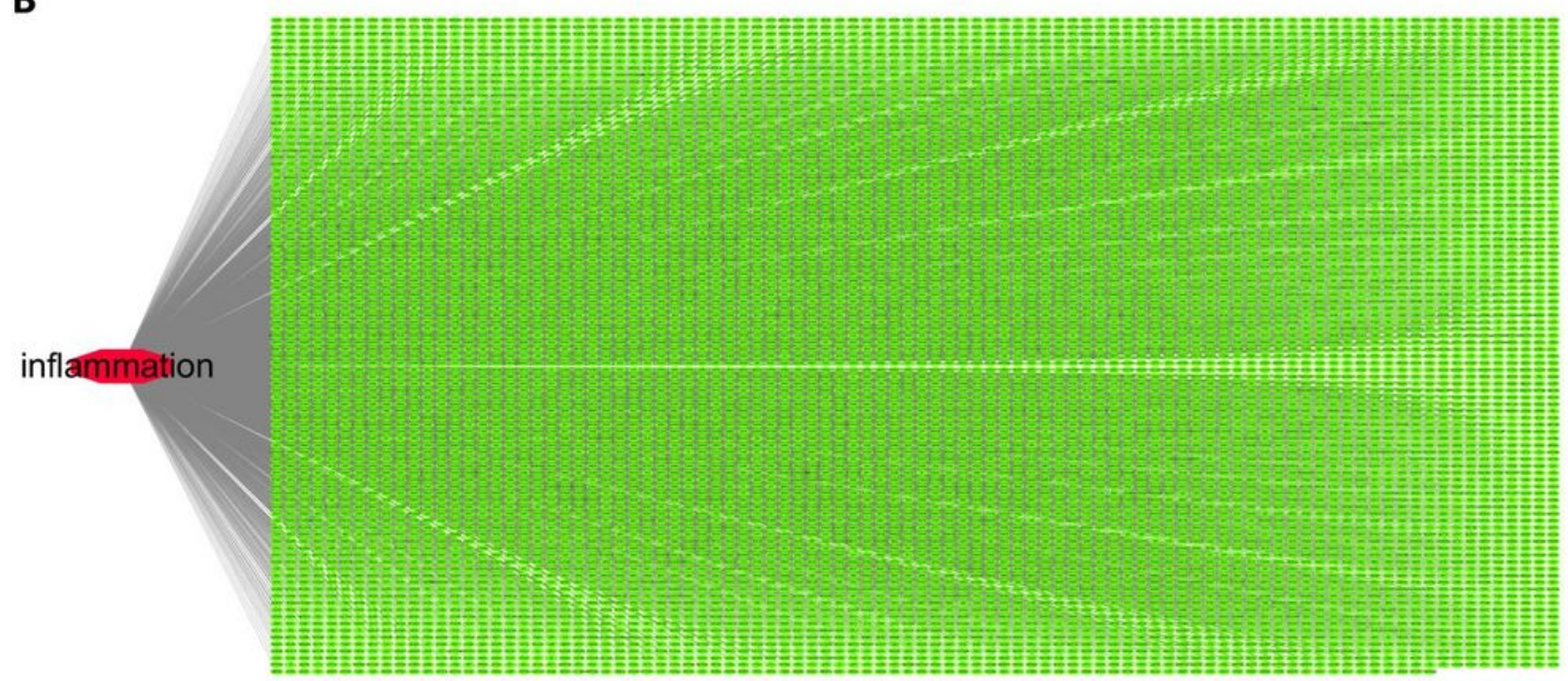

Figure 3

Target genes related to luteolin and inflammation. (A) 280 luteolin-targeted genes were fished by PharmMapper server: the red polygons and green circles respectively represented luteolin and luteolintargeted genes. (B) 9015 inflammation-targeted genes were harvested by CTD and Genecards database: the red polygons and green circles respectively represented inflammation and inflammation-targeted genes. 
A inflammation-targeted genes

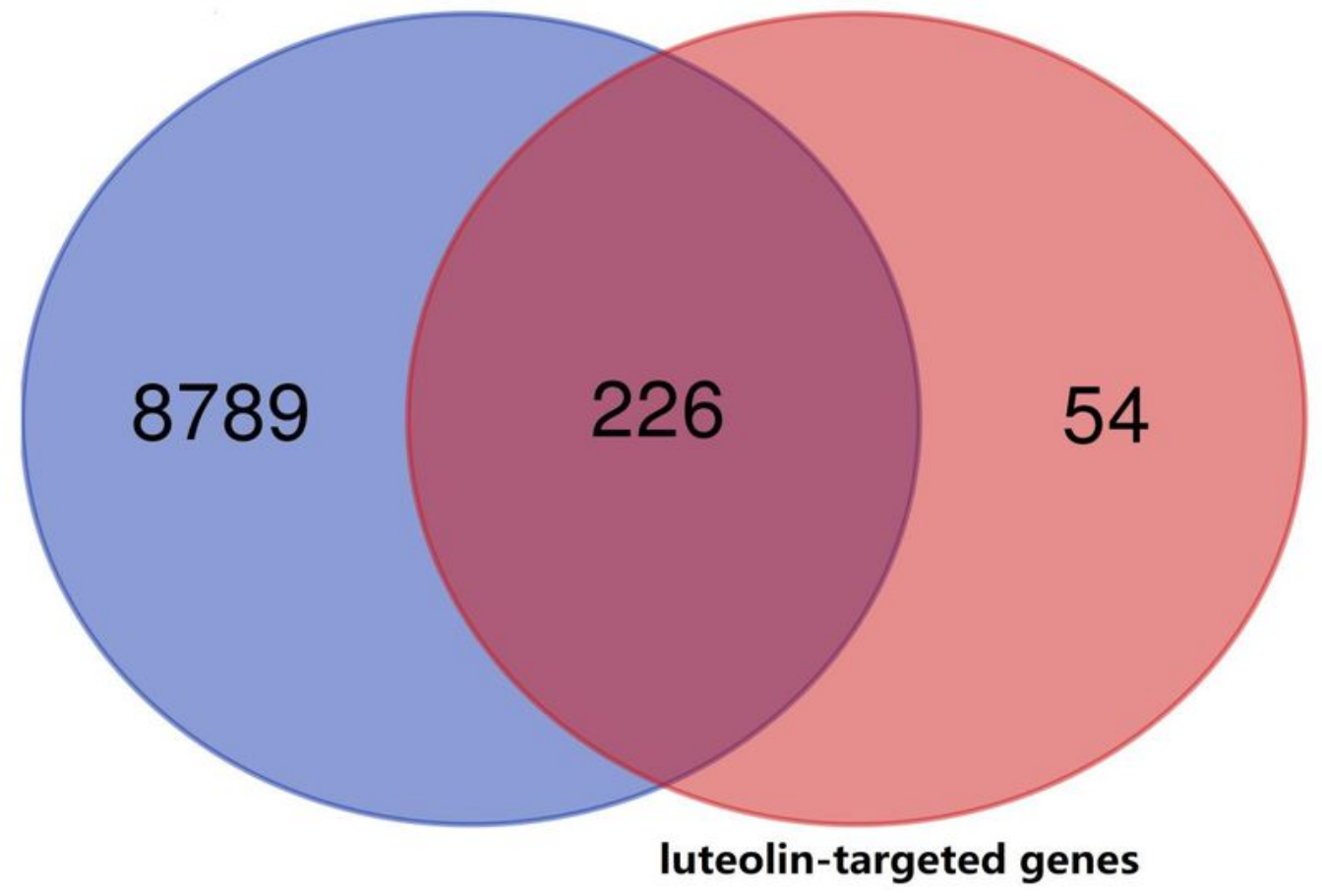

B

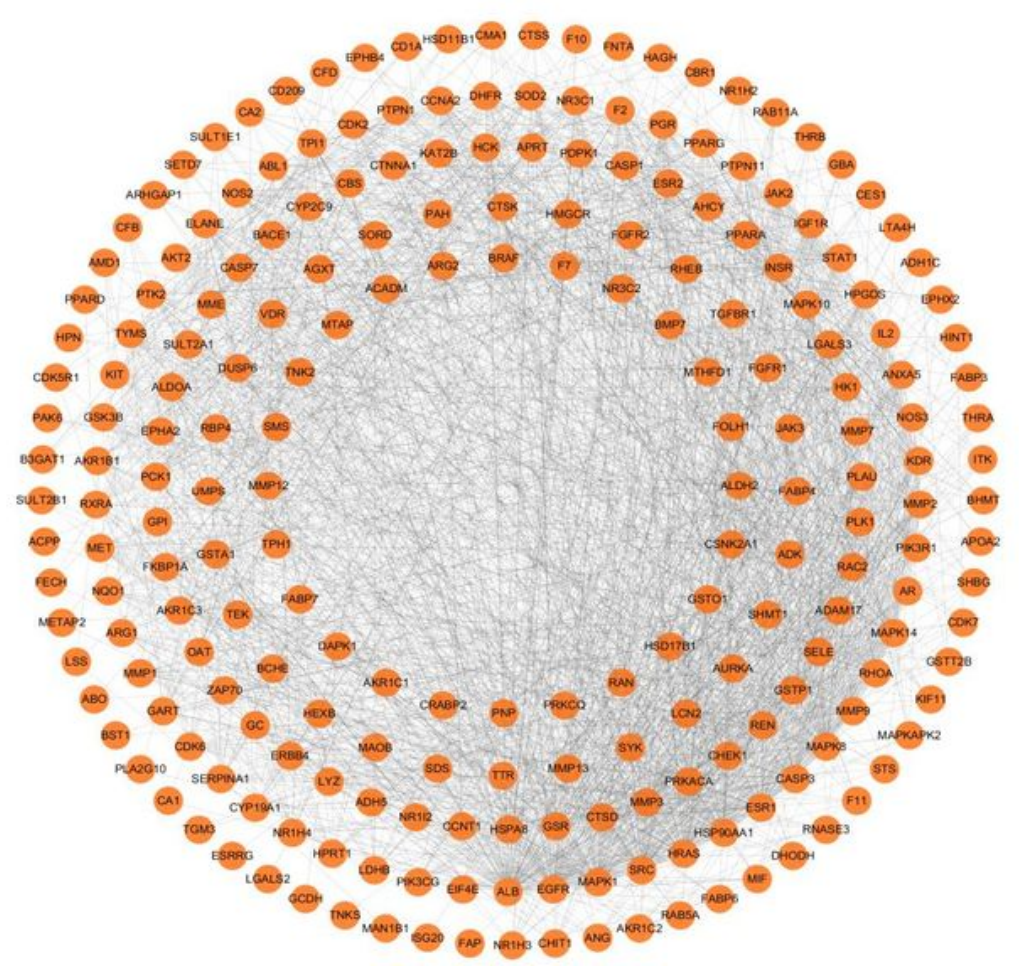

Figure 4

PPI network of luteolin against inflammation. (A) 226 Overlapping genes (target genes of luteolin against inflammation) between 280 luteolin-related genes and 9015 inflammation-related genes were analyzed by Draw Venn Diagram. (B) PPI network of luteolin against inflammation: 226 orange circles represented the targets of luteolin against inflammation and 2180 edges represented the interaction between targets. 
A
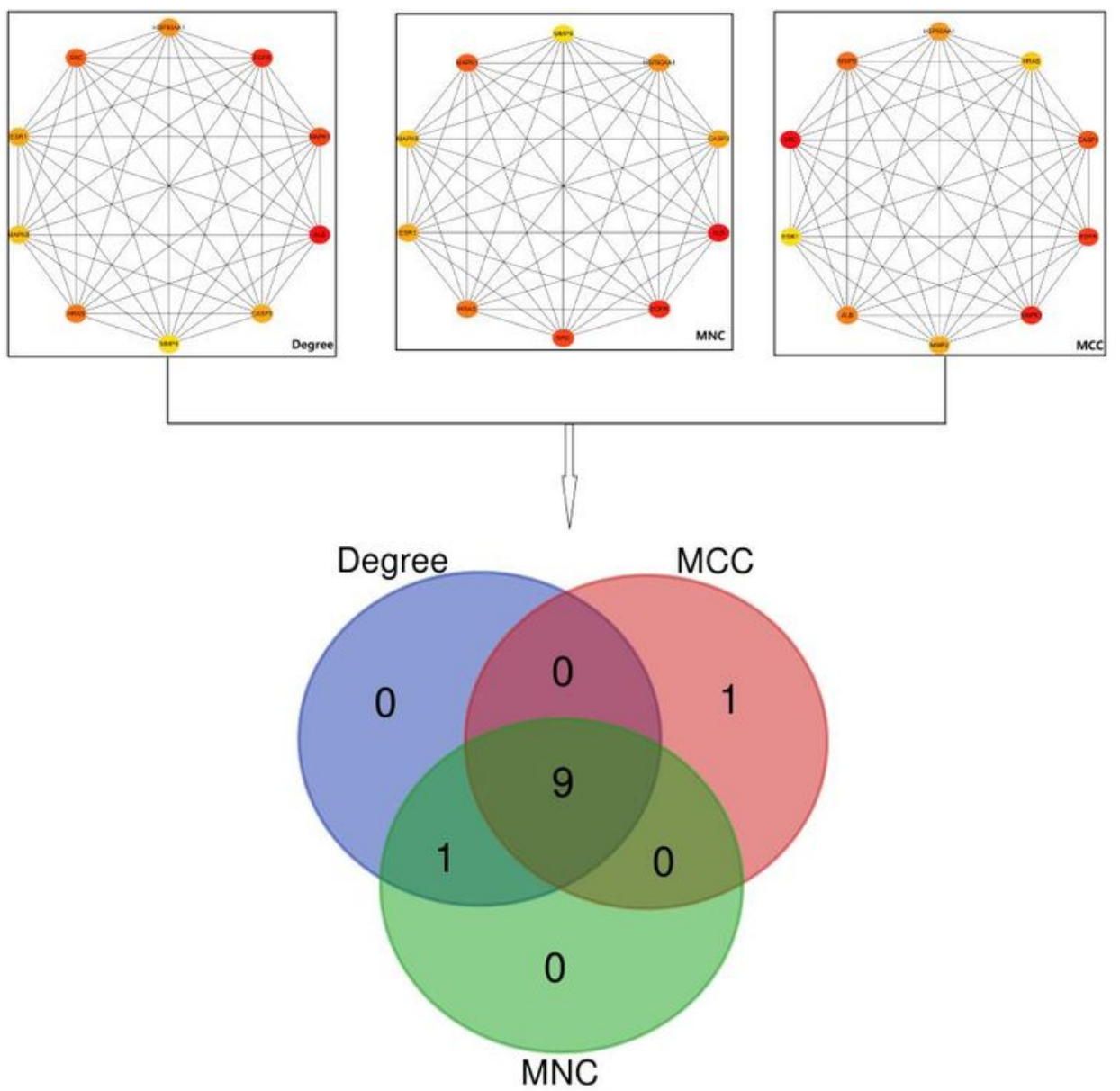

B

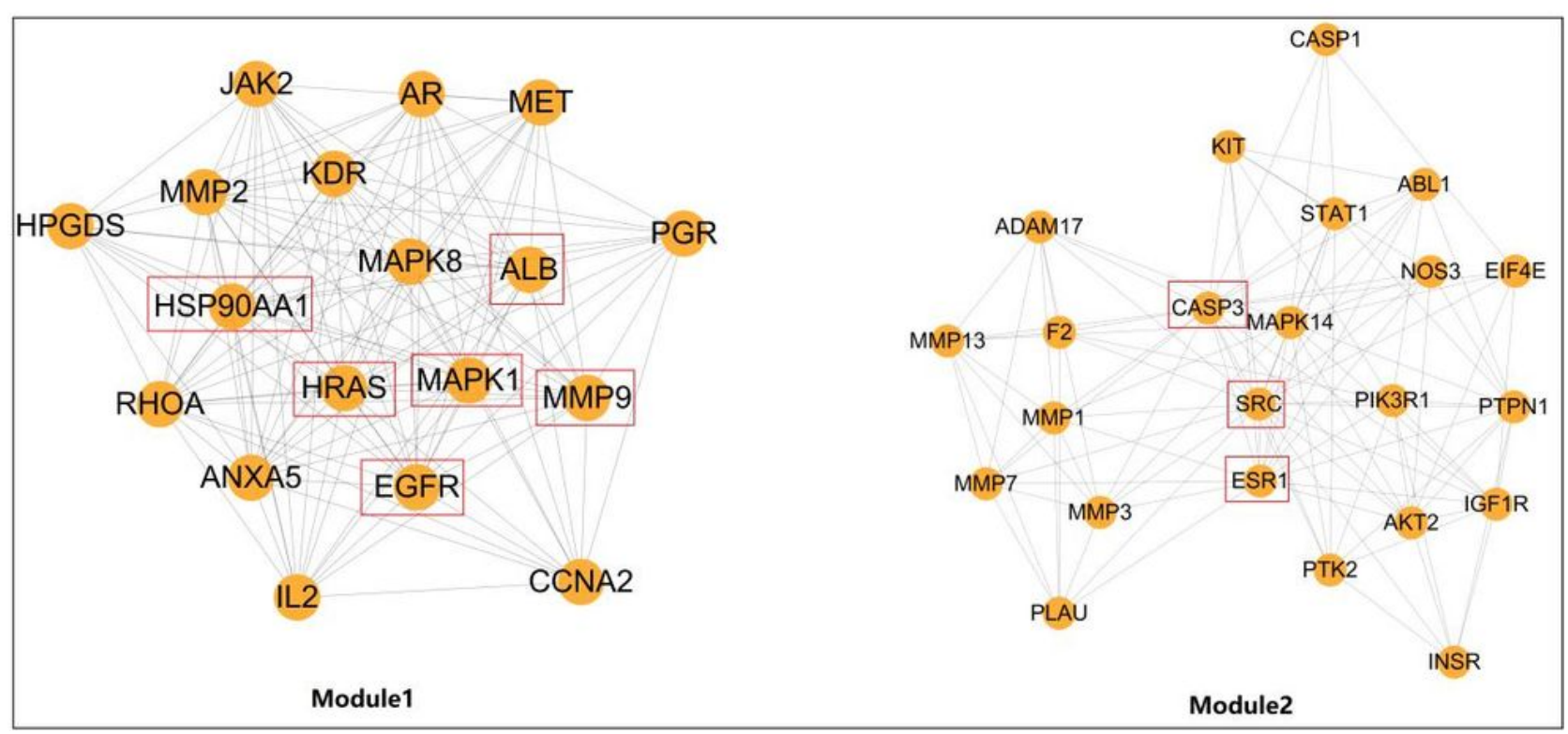

\section{Figure 5}

The core genes were analyzed by CytoHubba and MCODE. (A). PPI network were analyzed by CytoHubba ranked by degree, MNC and MCC, and then Draw Venn Diagram was used to get overlapping genes that represented the core genes. (B). The 2 most significant modules were analyzed by MCODE, and the circular nodes marked in red represented overlapping genes (core genes) contained in (A). 
A

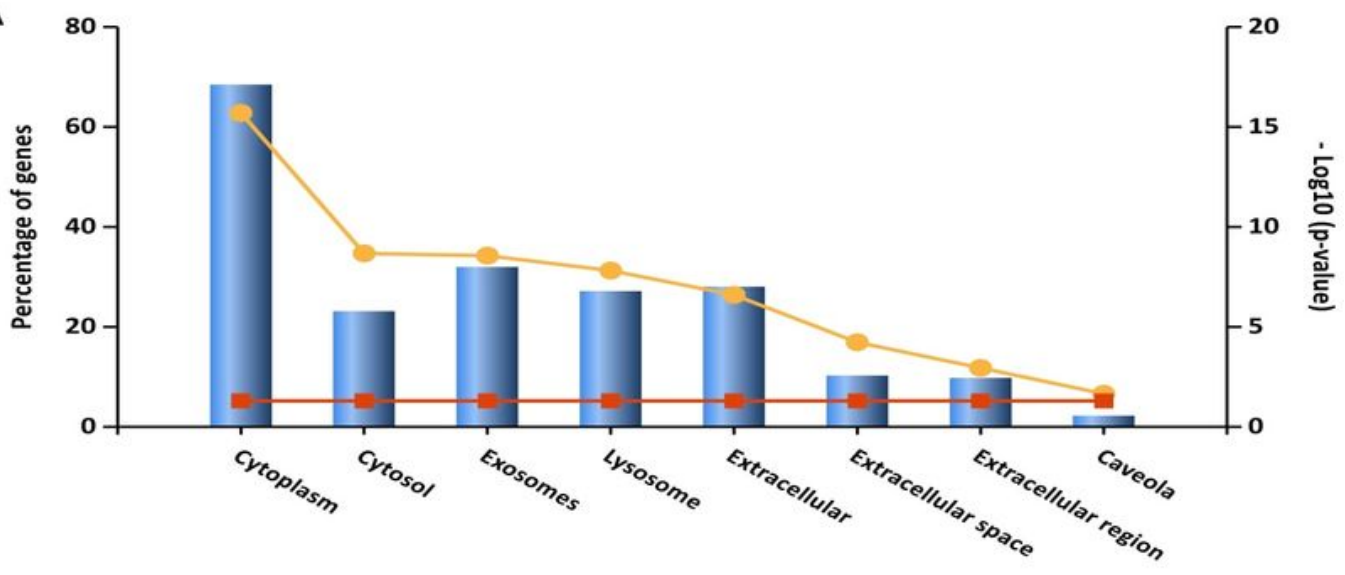

Cellular component

B

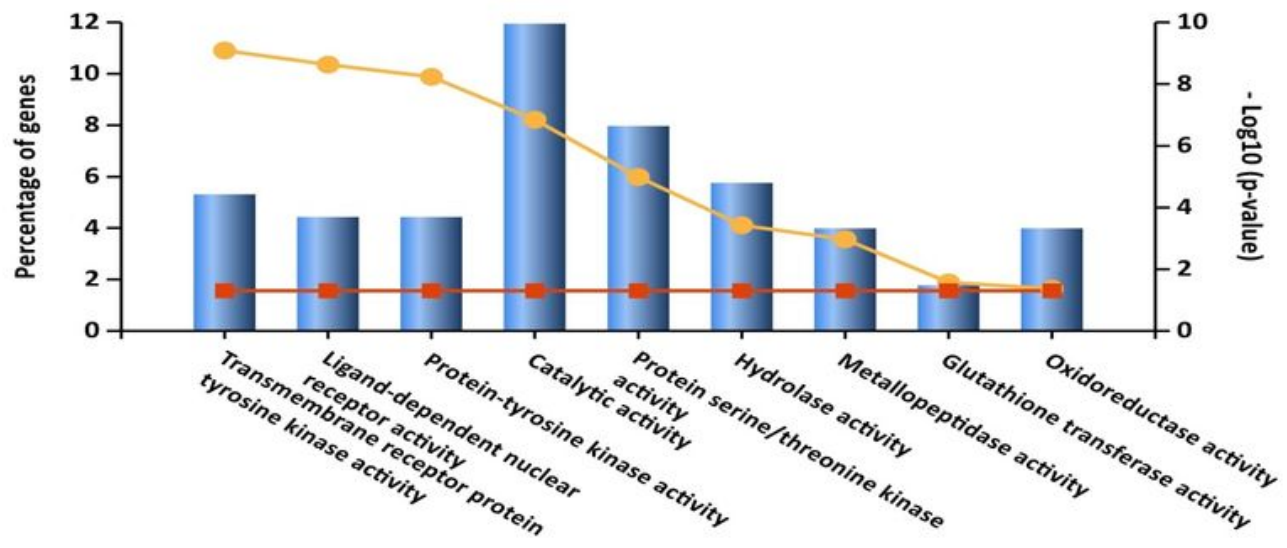

Molecular function

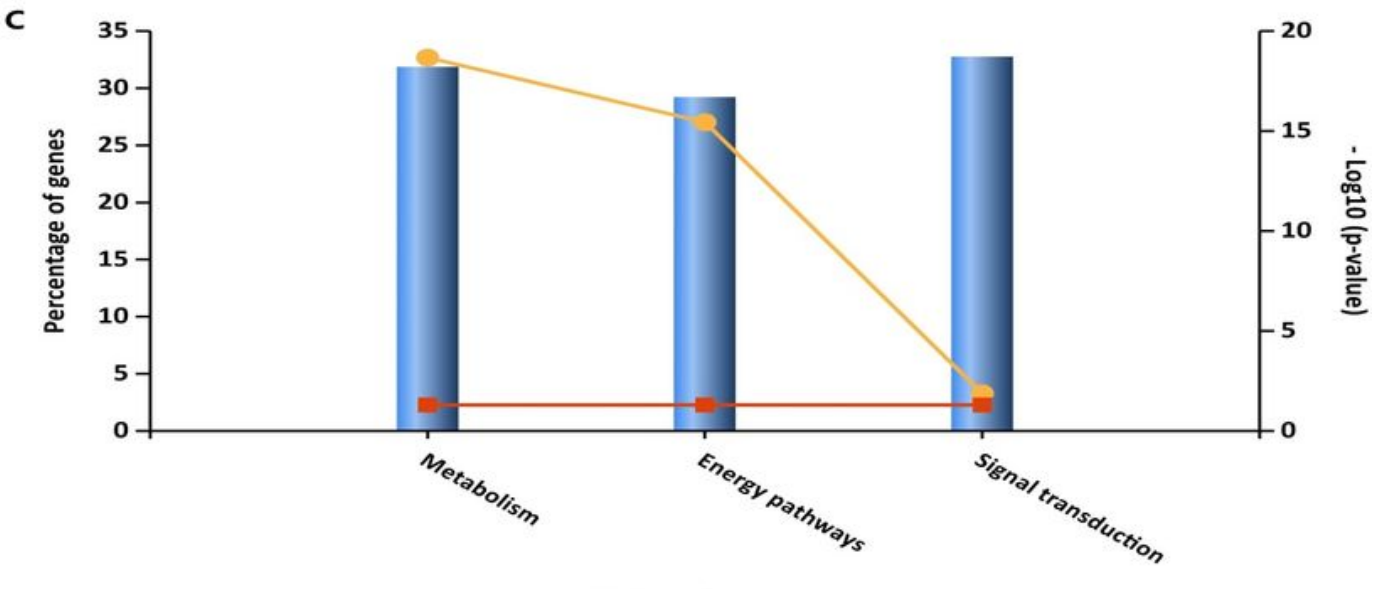

Biological process

\section{Figure 6}

GO analysis for targets of luteolin against inflammation. (A) 8 enriched CC items for targets of luteolin against inflammation; (B) 9 enriched MF items for targets of luteolin against inflammation; (C) 3 enriched BP items for targets of luteolin against inflammation. ( $p$ value $₫ 0.05$ were considered to be statistically significant for $\mathrm{GO}$ enrichment.) 
A Top 31 of KEGG Enrichment

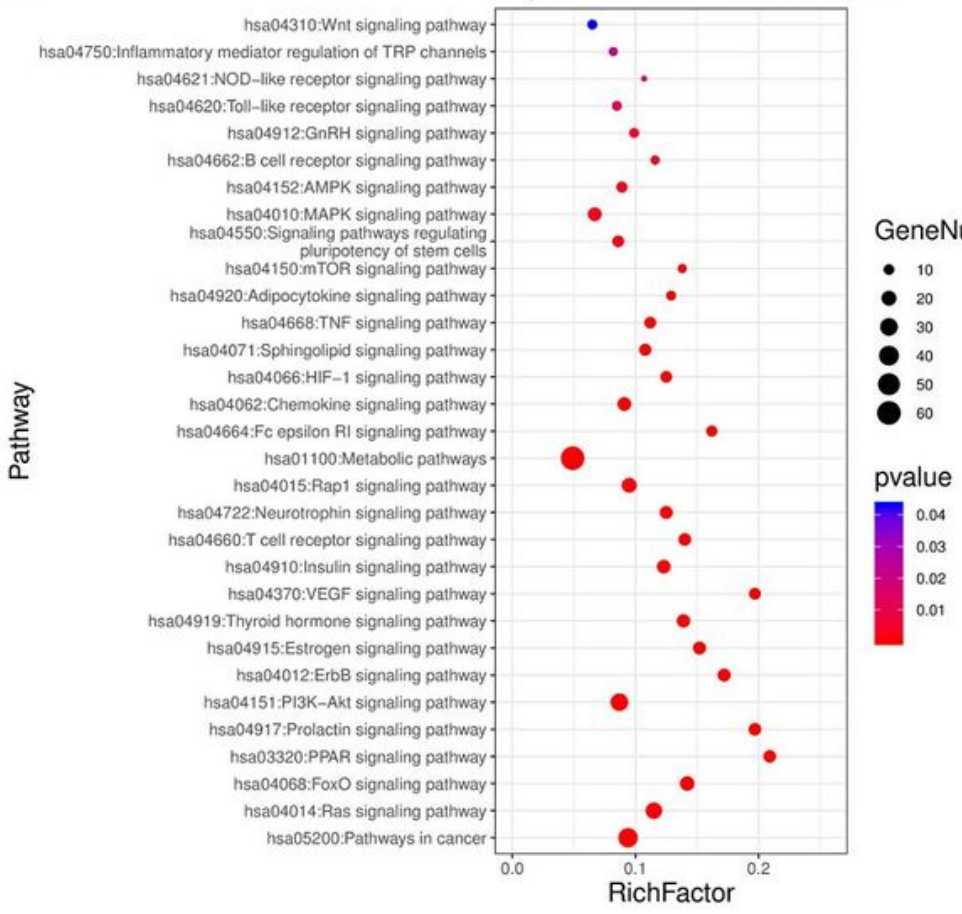

B

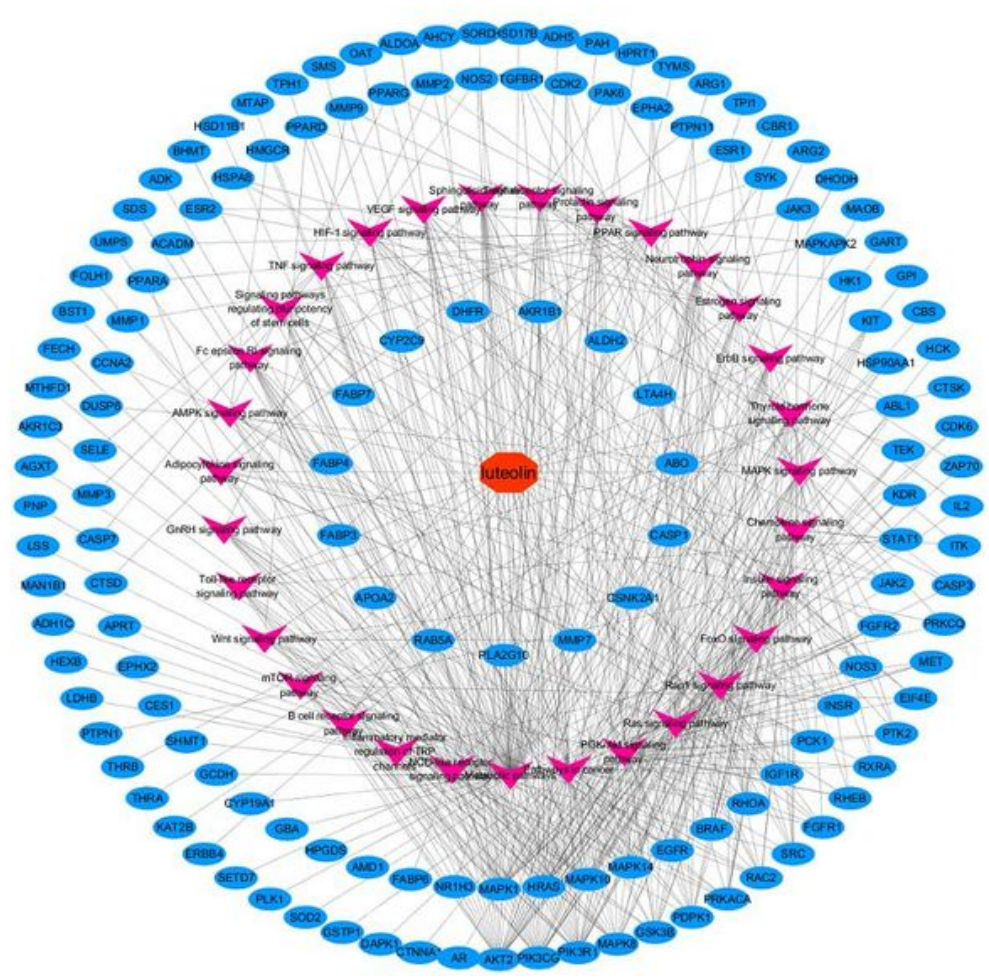

\section{Figure 7}

KEGG pathways of luteolin against inflammation. (A). KEGG enrichment was visualized by OmicShare: the gradual color represented the $P$ value and the size of the black spots represents the gene number enriched in the pathways. (B). Luteolin-target-pathway network: red polygons, red rrows and blue circles respectively represented luteolin, enriched genes and pathways. 
A

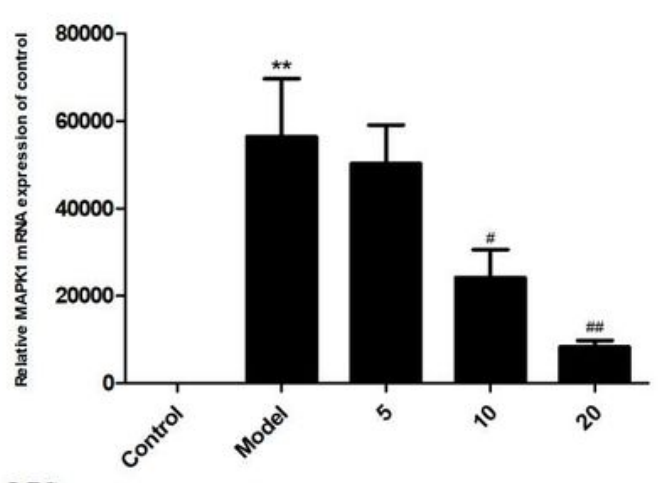

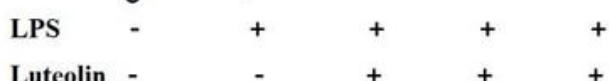

$(\mu \mathrm{M})$

C

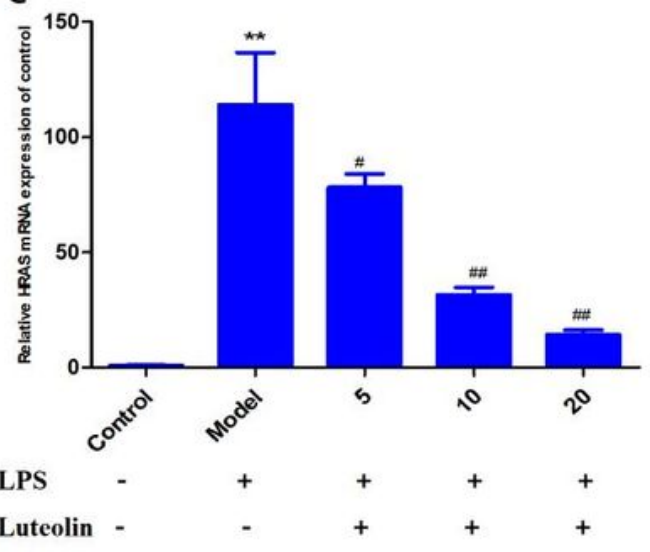

$(\mu \mathrm{M})$

E

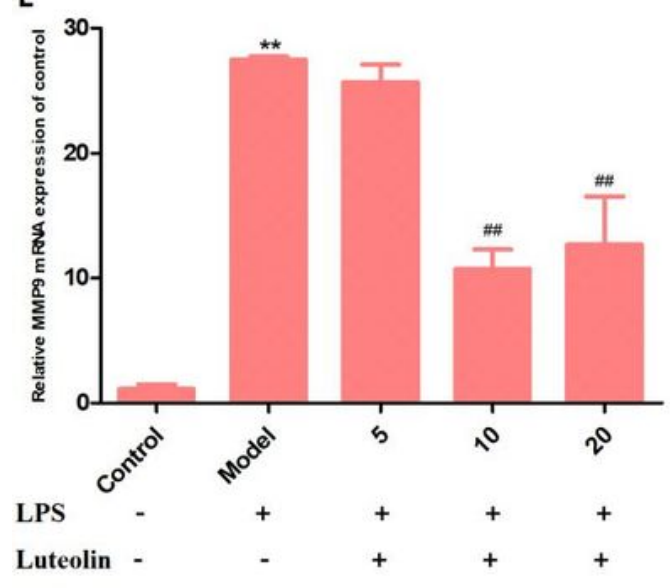

$(\mu \mathrm{M})$
B

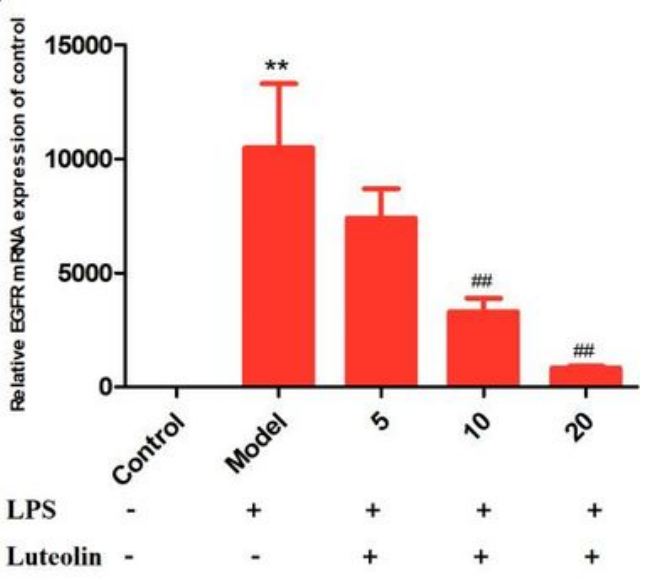

$(\mu \mathrm{M})$

D

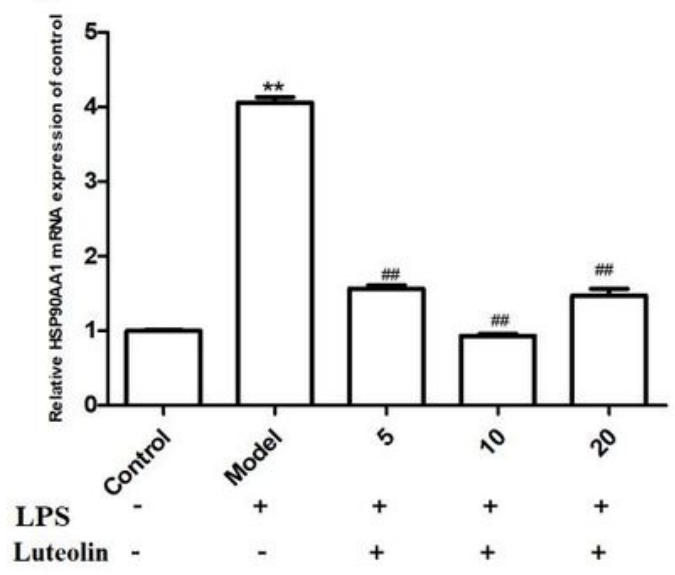

$(\mu \mathrm{M})$

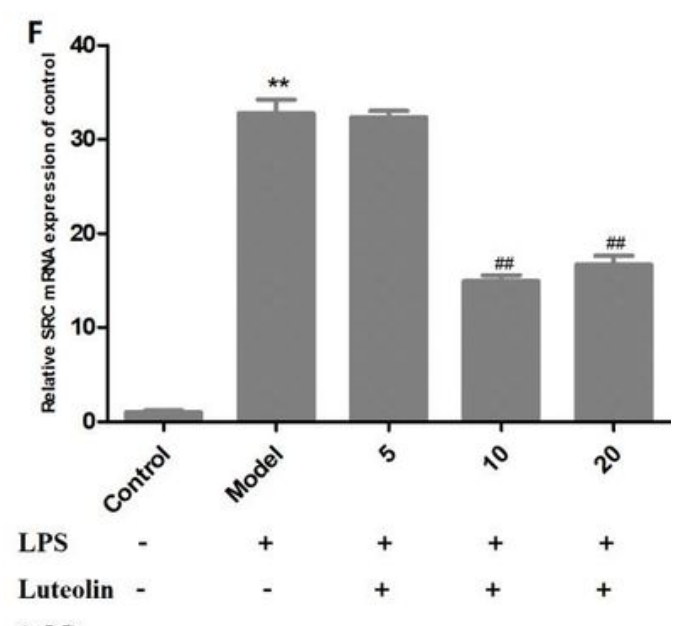

$(\mu \mathrm{M})$

\section{Figure 8}

RT-qPCR proved that luteolin inhibited LPS-induced overexpression of MAPK1, EGFR, HRAS, HSP90AA1, MMP9 and SRC mRNA. (A, B, C, D, E and F). Expression of MAPK1, EGFR, HRAS, HSP90AA1, MMP9 and SRC mRNA significantly increased after stimulation with LPS $(100 \mathrm{ng} / \mathrm{mL})$ for $24 \mathrm{~h}$, while luteolin decreased LPS-induced overexpression of MAPK1, EGFR, HRAS, HSP90AA1, MMP9 and SRC mRNA. 
(** $\mathrm{P}<0.01$ vs control group, \#P<0.05 vs model group, \#\#P $<0.01$ vs model group, the data were represent as the Mean \pm SD of three independent experiments.)

A

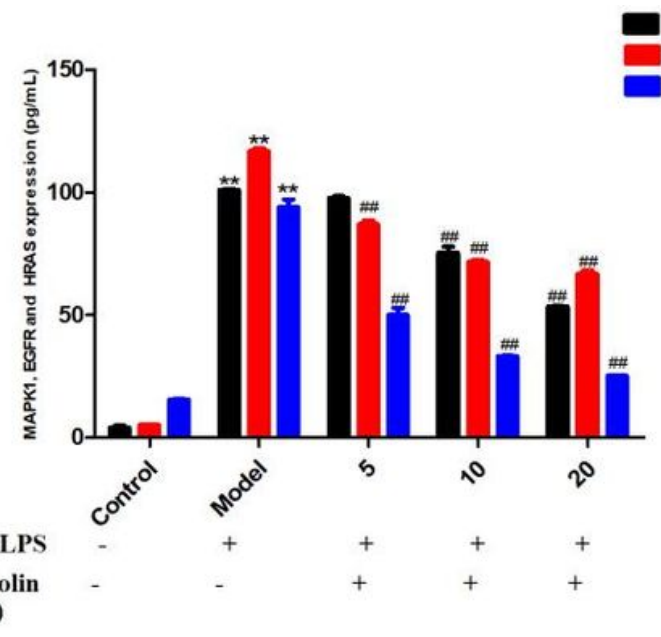

B

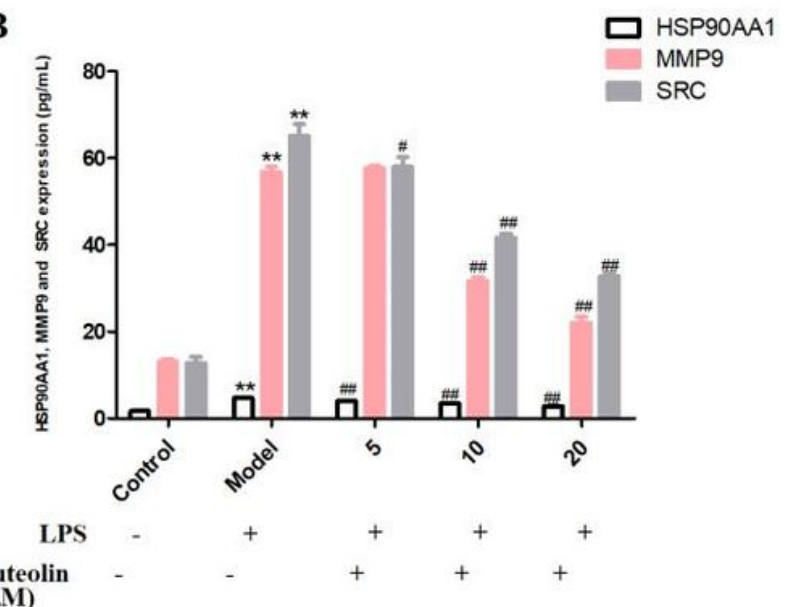

Figure 9

ELISA confirmed that luteolin inhibited LPS-induced overexpression of MAPK1, EGFR, HRAS, HSP90AA1, MMP9 and SRC. (A and B). Expression of MAPK1, EGFR, HRAS, HSP90AA1, MMP9 and SRC significantly increased after stimulation with LPS $(100 \mathrm{ng} / \mathrm{mL})$ for $24 \mathrm{~h}$, while luteolin decreased LPS-induced overexpression of MAPK1, EGFR, HRAS, HSP90AA1, MMP9 and SRC. ( ${ }^{\star *} \mathrm{P}<0.01$ vs control group, $\# \mathrm{P}<0.05$ vs model group, \#\#P $<0.01$ vs model group, the data were represent as the Mean \pm SD of three independent experiments.) 